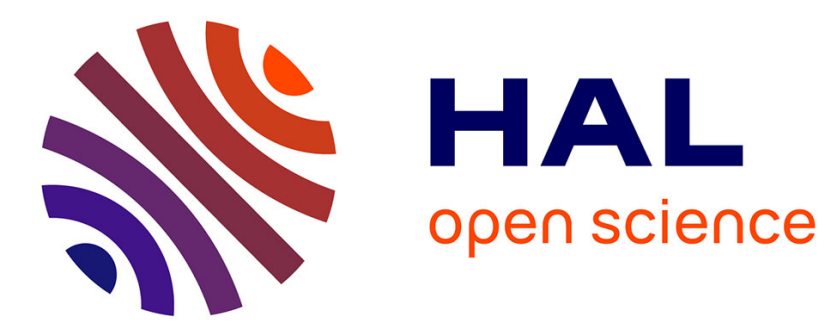

\title{
Wireless Network Performance Evaluation for Networked Robots
}

\author{
Insaf Sassi, Alexia Gouin, Jean-Marc Thiriet
}

\section{To cite this version:}

Insaf Sassi, Alexia Gouin, Jean-Marc Thiriet. Wireless Network Performance Evaluation for Networked Robots. ETFA 2017 - 22nd IEEE International Conference on Emerging Technologies and Factory Automation, Sep 2017, Limassol, Cyprus. hal-01665237

\section{HAL Id: hal-01665237 https://hal.science/hal-01665237}

Submitted on 8 Jan 2018

HAL is a multi-disciplinary open access archive for the deposit and dissemination of scientific research documents, whether they are published or not. The documents may come from teaching and research institutions in France or abroad, or from public or private research centers.
L'archive ouverte pluridisciplinaire HAL, est destinée au dépôt et à la diffusion de documents scientifiques de niveau recherche, publiés ou non, émanant des établissements d'enseignement et de recherche français ou étrangers, des laboratoires publics ou privés. 


\title{
Wireless Network Performance Evaluation for Networked Robots
}

\author{
Insaf Sassi, Alexia Gouin, Jean-Marc Thiriet \\ Univ. Grenoble Alpes, CNRS, GIPSA-Lab, F-38000 Grenoble, France \\ Email: firstname.name@gipsa-lab.grenoble-inp.fr
}

\begin{abstract}
The integration of the wireless network into the control loop of Networked Control Systems NCSs, given the stochastic behavior of wireless communication, can degrade the system performance i.e, its Quality of Control (QoC). It is necessary to study the implications of the communication constraints because control systems need a perfect communication medium. The performance of wireless network and its Quality of Service (QoS) must thus be analyzed in order to guarantee a good QoC. The present paper proposes an analysis of QoS performance metrics for networked systems (networked robots) using an analytic model based on Markov chain theory. The Markov model describes the IEEE 802.11 Distributed Coordination Function (DCF) for periodic round trip traffic of Wireless Networked Control System (WNCS).
\end{abstract}

\section{INTRODUCTION}

Wireless Networked Control Systems (WNCSs) are networked systems where a wireless network is integrated into the control loop. Wireless networks have many advantages such as flexibility, lack of wiring, mobility and fast deployment [5]. However, it is difficult to ensure correct and complete data transmission between the control loop components because of the stochasticity of the wireless network. This nondeterminism leads to problems that affect system performance. A diagnosis method is essential to monitor, diagnose and maintain the networked system in an operational state, hence the importance of the Bayesian Network (BN) for diagnosis of wireless networked mobile robot proposed in our work [7]. This $\mathrm{BN}$ for diagnosis permits to determine the cause of Quality of Control (QoC) degradation. The QoC is affected by the network state and specifically by its Quality of Service (QoS). When the network cannot ensure the required QoS, it is considered failing and so the QoC. This influence is due to delay, packet loss, jitter and throughput [8]. QoS requirements must be thus defined with respect to these parameters to guarantee a good system performance and then integrated into our bayesian model [7]. Wireless local area networks (Wlans) with IEEE 802.11 standard have been used in many Networked control systems as a wireless network architecture. The basic medium access control MAC for IEEE 802.11 is the Distributed Coordination Function (DCF) which is widely implemented in wireless products. It is a random access mechanism based on the carrier sense multiple access with collision avoidance CSMA/CA. Each station, having a frame to transmit, senses the medium to ascertain whether the wireless medium is busy or idle. If the medium is idle, the station transmits its packet. Otherwise, it defers transmission until the medium is sensed free without interruption for a period of time equal to a Distributed Inter-Frame Space (DIFS). The station is allowed to transmit when the backoff timer reaches zero. At each packet transmission, the backoff time is uniformly chosen in the range $[0 ; C W-1]$, where $\mathrm{CW}$ (Contention Window) is the current backoff window size. At the first transmission attempt, CW is equal to the initial backoff window size $C W_{\text {min }}$. After each unsuccessful transmission, $\mathrm{CW}$ is doubled until a maximum backoff size $C W_{\max }$ is reached. When the retry limit is reached, the packet is discarded. The verification of a successful data reception or the collision detection is done by the reception of an acknowledgment (ACK packet) from the destination station after a Short Inter-Frame Space (SIFS) time period from the reception of the data packet. In this work, the DCF performance is studied for a WNCS traffic in order to derive the required QoS parameters. Existing studies on the performance of 802.11 MAC DCF have focused on network capacity under saturated conditions using Markov chain theory to analyze its parameters. Bianchi in [1] developed a markov chain model to evaluate DCF performance (saturation throughput) under saturation conditions i.e, each node always has a packet to be transmitted (non empty queue). Many extensions of his work have been established and corrections have been introduced to conform the DCF specification specially for the backoff freezing mechanism [11]. [4] proved then the inaccuracy of Markov models based on Antonakopoulos work. The goal of our study is to adjust Bianchi Markov model to represent the behavior of periodic round trip traffic of a control loop under unsaturated conditions (the transmitting node does not always have a packet for transmission). Network performance for WNCS are derived and the smallest sampling period to maximize the channel bandwidth and to satisfy the QoS constraints is analytically determined. The paper is organized as follows. Section 2 is devoted to define the real time constraints in WNCS. Section 3 describes the Markov chain modeling of the round trip traffic of each control loop and the analytic analysis of QoS parameters. The developed analytic expressions of QoS parameters are studied in section 4. Finally, conclusions and perspectives are given in section 5 .

\section{REAL TIME CONSTRAINTS FOR NETWORKED SYSTEMS}

The network traffic in WNCSs has different requirements and characteristics compared to the best-effort network services. WNCSs, as real time systems, need a certain level 
of QoS in order to guarantee a good performance. The real time traffic conditions impose bounds on QoS metrics: bandwidth, delay, jitter and packet loss rate. In a network based WNCS, a sensor node periodically sends the sensor packets to a controller node. Once the controller receives the sensor packets, it computes the control law. Then, it creates and sends back control packets to the actuator node. The actuator and the sensor nodes are generally installed in one system which is a networked mobile robot in our case study. If we consider a WNCS with one controller and $\mathrm{n}$ mobile robots (stations), the network scenario consists of periodic round trip traffics representing control loops data flows. Each control loop implemented via the wireless network is characterized by a sampling period denoted $T$. The packets transmitted in the wireless network experience transmission delays. For WNCSs with feedback control, round trip traffic time $T_{R T T}$ is considered to analyze the system real time performance. It is the delay from the time the sensor sends the sensor packet to the time the actuator receives the control packet. In order to satisfy real time requirements (deadline requirements in WNCSs), $T_{R T T}$ is bounded by the control loop period $T$ :

$$
T_{R T T} \leq T
$$

Reducing the control loop sampling period $T$ improves the QoC. However, a smaller sampling period produces overload traffic in the network which causes longer transmission delays and consequently a degraded QoS. Such a degradation limits the system performance in term of QoC [6]. The minimal value of the sampling period that the network can tolerate is defined by analyzing the network dynamic and statistics. Its analytic expression defined in [6] and [3] does not represent the wireless network indeterminism. In this work, we present a stochastic method to determine the analytic expression of the smallest sampling period. Choosing $T$ is an important task to find a trade-off between the control loop performance and the offered resources in terms of network load as proved by [3] and [8]. The value of $T$ cannot go under a critical value defined as the real time requirement in equation (1). In this case, the network throughput reaches its maximum achievable value which is the saturation throughput $S$.

\section{QOS PERFORMANCE ANALYSIS FOR WIRELESS NETWORKED CONTROL SYSTEMS}

The objective of the proposed model is to determine the DCF performance for a WNCS with a periodic round trip traffic and to define the critical value of the sampling period $T$, the saturation throughput $S$ and the average round trip delay $T_{\text {avgRTT }}$. A Wlan of $\mathrm{n}$ closed control loops having the same transmission period $T$ is considered. In this case, the WNCS is composed of one controller and $n$ mobile robots equipped with sensors and actuators. The $n$ closed loops share the same communication medium, each has two types of packets to be transmitted: the sensor and the control packets. Our proposed model corresponds to each control loop traffic in the shared medium. It extends the Bianchi model in [1] by adding an empty queue stage (model of the queue stage is proposed in
[9]) to represent the unsaturated conditions since the robot does not have always a packet to be transmitted. In fact, the sensor task in the robot provides the information about the system output (the robot position and orientation) every sampling period only. Our contribution consists in adding another backoff stage for the control packet which is triggered if the sensor packet is received. Adding this second backoff stage allows studying the round trip real time traffic. Modeling the backoff freezing has been adopted in many extensions of the Bianchi model. [10] explained that those models are inaccurate and proved that the Bianchi model is more effective in capturing the actual protocol behavior using a simpler model and providing accurate results. For these reasons, the backoff freezing is not presented in our Markov model to simplify the performance analysis.

\section{A. IEEE 802.11 DCF analytical model for WNCS}

The developed Markov model in figure 1 represents the behavior of each control loop in the network assuming to operate in ideal channel conditions (no signals interference and no hidden terminals). In this case, any transmission error is due to packets collisions. Let $\mathrm{p}$ denotes the collision probability of a transmitted packet. This probability $\mathrm{p}$ is independent of the backoff procedure, and the backoff counter is decremented at the end of the backoff slot as explained in [1]. The state of each packet to be transmitted is described by $j, k$, where $j$ stands for the backoff stage and $k$ represents the backoff counter which takes value in $\left[0, W_{j}-1\right]$ if $j \in[0,2 R+1]$. The random access in a Wlan, as described in section 2, is represented by two stochastic processes. Let $b(t)$ be the random process representing the backoff counter value at a time slot t and let $s(t)$ be the random process for the backoff stage $j$ where $j \in[0,2 R+1]$. The random process $b(t)$ is uniformly distributed in $\left[0 ; W_{j}-1\right]$ in the $j^{\text {th }}$ backoff period if $j \in[0,2 R+1]$. The discrete time Markov chain of figure 1 depicts the bi-dimensional process $\{s(t), b(t)\}$ for both sensor and control packets of each control loop. We add an empty queue stage to the Bianchi model represented by stage $j=-1$. After the backoff stage of the control packet, we have $\mathrm{N}$ queue states $(-1, \mathrm{k})$ where $k \in[0 ; N-1]$ representing the remaining time before starting access to the communication channel. The $\mathrm{n}$ communicating nodes are operating in discrete time. Let $T_{\text {slot }}$ denotes the duration of a time slot. Therefore, the number $N$ of empty queue states is defined as follows as explained in [9]:

$$
N=\frac{T-T_{\min R T T}}{T_{\text {slot }}}
$$

where $T_{\min R T T}$ is the minimum round trip delay.

The empty queue state is determined by the actual round trip time which is defined in $\left[T_{\operatorname{minRTT}}+i T_{\text {slot }}, T_{\operatorname{minRTT}}+(i+\right.$ 1) $\left.T_{\text {slot }}\right]$ in the current control period where $i \in[0, N-1]$. Consequently, $q_{k}=1$ holds only for $k=i$ otherwise $q_{k}=0$ where $q_{k}$ is the probability to be in one of the $N$ empty queue states. Let $R$ be the maximum value of retransmission. The state transition diagram of the Markov chain model of figure 1 has the following transition probabilities: 


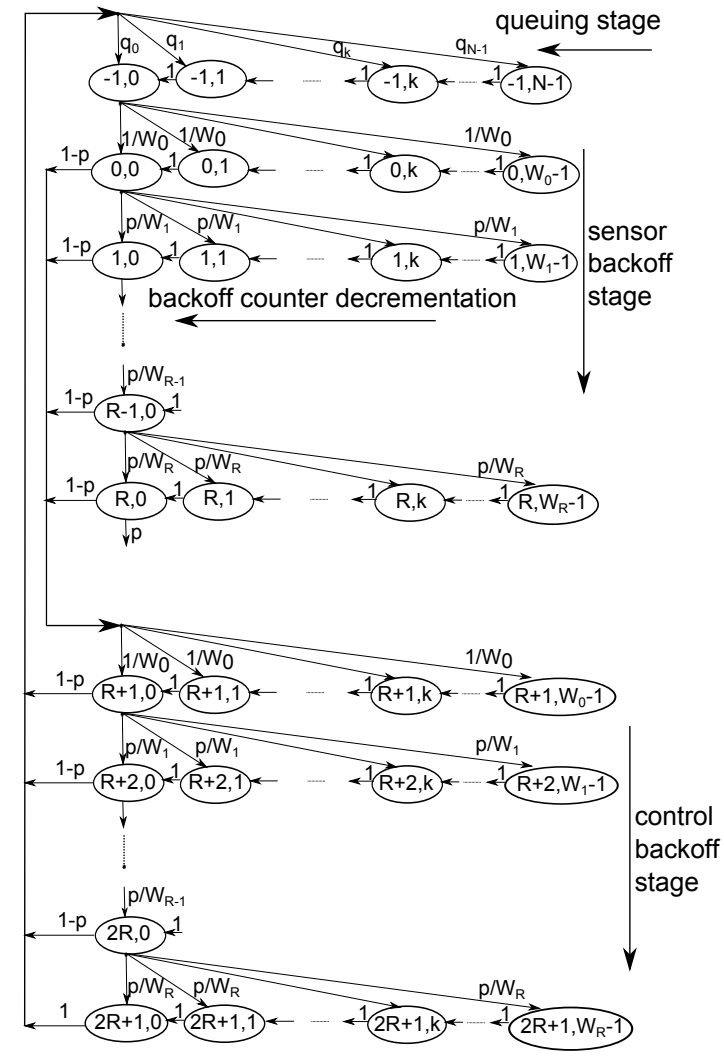

Fig. 1. Markov chain model for data transmissions in a network of networked mobile robots

1) A new sensor packet following a successful transmission of both closed loop packets starts with a backoff stage 0 , where the backoff value is uniformly chosen in range $\left[0 ; W_{0}-1\right]$ :

$P[(0, k) \mid(j, 0)]=\frac{1-p}{W_{0}} ; R+1 \leq j<2 R+1 ; k \in$ $\left[0, W_{0}-1\right]$

2) The backoff stage of the control packet reaches the value $2 R+1$, it is then reset to its initial state whatever the control packet transmission state is (successful or collision):

$P[(0, k) \mid(2 R+1,0)]=\frac{1}{W_{0}} ; 0 \leq k \leq W_{0}-1$

3 ) In the beginning of each slot time, the backoff time is decremented:

$P[(j, k) \mid(j, k+1)]=1 ; 0 \leq j \leq 2 R+1 ; 0 \leq k \leq W_{j}-2$

4) When an unsuccessful transmission occurs at backoff stage $j-1$, the backoff stage is incremented and the new backoff time value is uniformly chosen in $\left[0 ; W_{j}-1\right]$ : $P[(j, k) \mid(j-1,0)]=\frac{p}{W_{j}} ; 0 \leq k \leq W_{j}-1 ; 1 \leq j \leq R$ ou $R+2 \leq j \leq 2 R+1$

5) Before starting a new control period, a countdown is performed until the arrival of a new frame at the networked robot queue:

$$
P[(-1, k) \mid(-1, k+1)]=1 ; 0 \leq k \leq N-2
$$

The stationary distribution of the Markov chain is $b_{j, k}=$ $\lim _{t \rightarrow+\infty} \operatorname{Pr}\{s(t)=j, b(t)=k\}$. The following relations are defined through chain regularities:

$$
\begin{gathered}
b_{-1,0}=b_{0,0} \\
b_{j, 0}=p^{j} b_{0,0} ; 0 \leq j \leq R \\
b_{j, 0}=p^{j-(R+1)} b_{R+1,0} \\
=p^{j-(R+1)}(1-p) \sum_{i=0}^{R} b_{i, 0} \\
=p^{j-(R+1)}\left(1-p^{R+1}\right) b_{0,0} \\
R+1<j \leq 2 R+1 \\
b_{j, k}=\frac{W_{j}-k}{W_{j}} b_{j, 0} \\
0 \leq j \leq 2 R+1 \\
1 \leq k \leq W_{j}-1
\end{gathered}
$$

If we assume that $T_{R T T} \in\left[T_{\min R T T}, T\right]$ follows a Poisson distribution with parameter $\lambda$ :

$$
\begin{aligned}
P\left(T_{\operatorname{minRTT}}+i T_{\text {slot }} \leq T_{R T T}\right. & \left.<T_{\operatorname{minRTT}}+(i+1) T_{\text {slot }}\right) \\
= & \frac{\lambda^{i}}{i !} \mathrm{e}^{-\lambda}, i \in[0, N-1]
\end{aligned}
$$

Consequently, the probability that the actual round trip delay to be in the $i^{t h}$ slot in $\left[T_{\min R T T}, T\right]$ is equal to $\frac{\lambda^{i}}{i !} \mathrm{e}^{-\lambda}$ and the states in the empty queue stage are obtained as following as explained in [9]:

$$
b_{-1, k}=\left(\sum_{i=k}^{N-1} \frac{\lambda^{i}}{i !} \mathrm{e}^{-\lambda}\right) b_{0,0}
$$

By imposing the normalization condition, the following relation is obtained:

$$
\sum_{j=0}^{R} \sum_{k=0}^{W_{j}-1} b_{j, k}+\sum_{j=R+1}^{2 R+1} \sum_{k=0}^{W_{j}-1} b_{j, k}+\sum_{k=0}^{N-1} b_{-1, k}=1
$$

From equations (3), (4), (5), (6) , (8) and (9), we obtain the expression of $b_{0,0}$ :

$b_{0,0}=\left[\frac{2-p^{R+1}}{2}\left(\frac{1-p^{R+1}}{1-p}+\sum_{j=0}^{R} W_{j} p^{j}\right)+\sum_{k=0}^{N-1} \sum_{i=k}^{N-1} \frac{\lambda^{i}}{i !} \mathrm{e}^{-\lambda}\right]^{-1}$

As we study the round trip traffic performance of each control loop, let $p_{\tau}$ be the probability that the control loop transmits in the backoff stage during a randomly chosen slot. A control loop transmits when the backoff counter (in the robot or in the controller side) reaches 0 . The probability $p_{\tau}$ has this following expression:

$$
p_{\tau}=\sum_{j=0}^{2 R+1} b_{j, 0}=\left(2-p^{R+1}\right) \frac{1-p^{R+1}}{1-p} b_{0,0}
$$

We can now express the collision probability $p$. It is the probability that at least one of $n-1$ remaining nodes transmits in a time slot. The $\mathrm{p}$ expression is given by:

$$
p=1-\left(1-p_{\tau}\right)^{n-1}
$$


TABLE I

NOTATIONS

\begin{tabular}{|c|c|}
\hline Notation & Explanation \\
\hline$E[P]$ & average packet payload size \\
$E[$ slot $]$ & average slot duration \\
$T_{H}$ & time duration to transmit a packet header \\
$T_{E[P]}$ & time duration to transmit the average payload \\
$T_{S I F S}$ & SIFS time \\
$T_{\text {Ack }}$ & time duration to transmit an ACK \\
$T_{D I F S}$ & DIFS time \\
$T_{\text {acktimeout }}$ & time required for an ACK timeout \\
\hline
\end{tabular}

Let $p_{s}$ stands for the probability of a successful transmission and $p_{b}$ denotes the probability that the channel is busy in a time slot. They are given by the following expressions as in [1]:

$$
\begin{gathered}
p_{s}=n p_{\tau}\left(1-p_{\tau}\right)^{n-1} \\
p_{b}=1-\left(1-p_{\tau}\right)^{n}
\end{gathered}
$$

The developed Markov model served as a tool to establish probabilities expressions of $\mathrm{p}, p_{\tau}, p_{s}$ and $p_{b}$. This will enable us to determine analytically the critical values of the sampling period $T$ that guarantees a good QoS performance and thus a good QoC. It also permits to define throughput and the average round trip delay analytic expressions in the next section.

\section{B. Saturation throughput and the critical sampling period}

The saturation throughput $S$ is the maximum achievable throughput under critical real time constraints. It is defined as the measure of information load delivered successfully per second. Let $T_{s}$ denotes the average time the channel is sensed busy due to a successful transmission and $T_{c o l}$ stands for the average time during which the channel is sensed busy because of a collision. $T_{s}$ and $T_{c o l}$ are calculated as following for the basic access mode:

$$
\begin{gathered}
T s=T_{H}+T_{E[P]}+T_{S I F S}+T_{A c k}+T_{D I F S} \\
T c o l=T_{H}+T_{E[P]}+T_{D I F S}+T_{\text {acktimeout }}
\end{gathered}
$$

where $E[P], T_{S I F S}, T_{A c k}, T_{D I F S}, T_{H}$ and $T_{\text {acktimeout }}$ are defined in table I.

We can express $\mathbf{S}$ as in [10]:

$$
\begin{aligned}
S & =\frac{p_{s} E[p]}{E[\text { slot }]} \\
& =\frac{p_{s} E[p]}{\left(1-p_{b}\right) T_{\text {slot }}+p_{s} T_{s}+\left(p_{b}-p_{s}\right) T_{\text {col }}}
\end{aligned}
$$

where $p_{s} T_{E[p]}$ denotes the average amount of payload information successfully transmitted in a time slot and $p_{b}-p_{s}$ is the probability that the channel contains a collision.

The $n$ control loops have the same sampling period. The critical real time transmission period $T_{c}$ that guarantees a satisfying QoS is defined as:

$$
T_{c}=\frac{2 n E[P]}{S}
$$

TABLE II

NETWORK PARAMETERS

\begin{tabular}{|c|c|}
\hline packet payload & 80 bytes \\
MAC header & 272 bits \\
Physical header & $192 \mathrm{bits}$ \\
ACK & 112 bits + PHY header \\
Channel bit rate & $11 \mathrm{Mbit} / \mathrm{s}$ \\
Slot Time & $20 \mu \mathrm{s}$ \\
$T_{S I F S}$ & $10 \mu \mathrm{s}$ \\
$T_{D I F S}$ & $50 \mu \mathrm{s}$ \\
$R$ & 7 \\
\hline
\end{tabular}

\section{Round trip delay analysis}

[2] analyzed the packet transmission delay as the time between the instant the packet is put into service (the packet is at the head of the MAC queue) and the instant of time the packet is successfully received at the destination node. The average time delay for a successfully transmitted sensor and control packets, which is the average round trip delay, is expressed as follows:

$$
T_{\text {avgRTT }}=2 E[X] * E[\text { slot }]
$$

where $E[X]$ is the average number of time slots required for a successfully transmitted packet and it is analyzed in [2]. The average round trip delay $T_{a v g R T T}$ is not only determined by equation (19) since $\lambda$ is still unknown. $T_{a v g R T T}$ has another expression from equation (7):

$$
T_{\text {avgRTT }}=T_{\operatorname{minRTT}}+\lambda T_{\text {slot }}
$$

where $T_{\min R T T}=2 * T_{s}$.

$T_{\text {avgRTT }}$ is thus given by solving equations (19) and (20).

\section{CASE STUDY}

We consider an IEEE $802.11 \mathrm{~b}$ Wlan with $n$ control loops: $\mathrm{n}$ robots and 1 access point where the controller is implemented (the robots in the lab are equipped with IEEE $802.11 \mathrm{~b}$ network interfaces). The network parameters values are defined in table II. We analyzed the analytic values of $p, S, T_{c}$ and $T_{\text {avgRTT }}$ for different minimum contention window sizes $C W_{\min }$ which corresponds to $W_{0}$ in our analysis in section 3 where $C W_{\min } \in\{4,8,16,32\}$. The minimum contention window size $C W_{\min }$ value impacts the collision probability. When the contention window size is small, the number of transmission attempts increases and so is the collisions rate specially for a large number of contending closed loops. Consequently, the saturation throughput $S$ corresponding to the smallest value of $C W_{\min }$ is the most penalized where the critical sampling period $T_{c}$ is the largest as shown in figures 2 and 3. Regarding the average delay, figure 4 shows that it increases when $C W_{\min }$ increases because the contention window size affects the waiting delay of the node attempting to transmit. The maximum achievable throughput $S$, theoretically determined by our analytic analysis and represented in figure 2 , depends on the number of contending control loops and the initial contention window $C W_{\min }$. In fact, if the contention window size $W$ increases in the case of a large $n$, the 


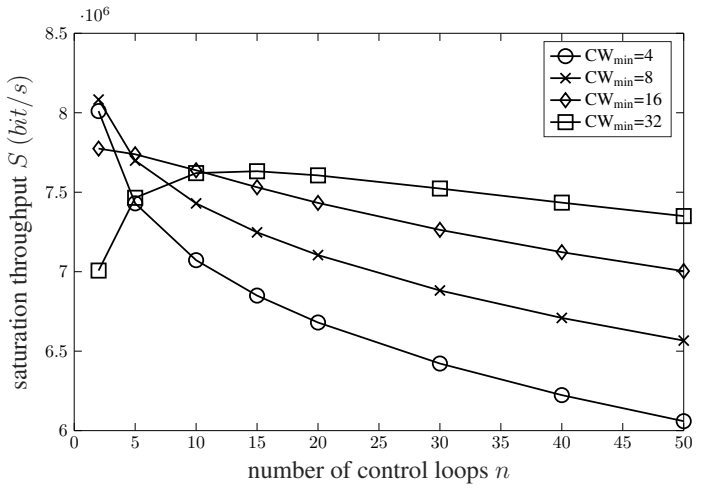

Fig. 2. Saturation throughput for different values of $C W_{\min }$

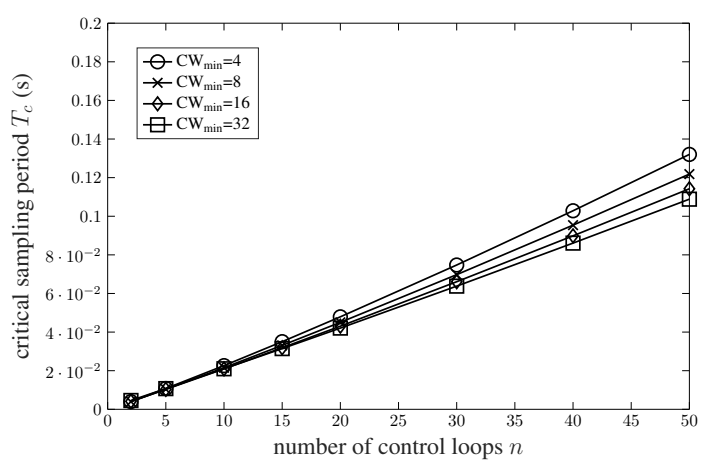

Fig. 3. Critical sampling period for different values of $C W_{\min }$

throughput becomes good, but it penalizes the throughput when $n$ is small. A large value of $C W$ causes throughput limitation for a small number of contending stations. The transmitting node waits for long time before attempting the transmission. Consequently, if the number of control loops is small and $C W$ is small, the number of idle slots is low which results in a good throughput. When the network size $n$ increases the throughput is lowered. Moreover, the critical sampling period of $T_{c}$ increases when $n$ increases in order to avoid QoS degradation. If $T$ is reduced below $T_{c}$ for every number of contending control loops, the QoS requirements are dissatisfied, causing network overload and longer delays, hence the control performance is degraded (QoC degradation). Figure 4 shows that when $n$ increases, the average round trip delay $T_{a v g R T T}$ increases as well. This proves that the increase of the number of contending control loops deteriorates the network performance.

\section{CONCLUSION}

The stochastic behavior of wireless networks introduces timing uncertainties which cause control performance degradation. These uncertainties and their impact on the QoC are modeled by a $\mathrm{BN}$ for diagnosis where we need to define the limit of the good performance based on $S, T_{c}$ and $T_{a v g R T T}$. Studying the network dynamics is thus an important task to define the QoS performances for a WNCS. An analytic Markov model is established characterizing the periodic round

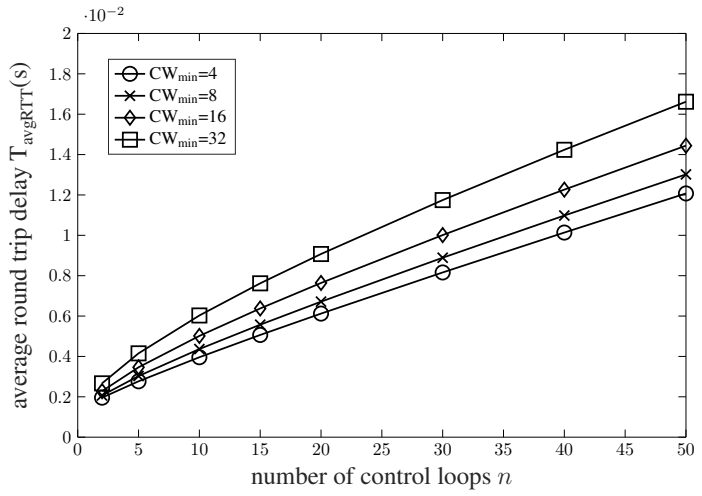

Fig. 4. Average round trip delay for different values of $C W_{\min }$

trip traffic behavior of each control loop. Thanks to our model, mathematical expressions are determined for the maximum achievable throughput and average delay from the established relations of the Markov chain stationary distribution. Moreover, the critical value of the sampling period below which the QoS requirements are not satisfied, is defined. However, Network analysis is not sufficient. Control performances should also be taken into consideration in the design step. Further work will propose a solution for the case of WNCSs with different sampling periods.

\section{REFERENCES}

[1] Bianchi, G. (2000). Performance analysis of the IEEE 802.11 distributed coordination function. IEEE Journal on selected areas in communications, 18(3), 535-547.

[2] Chatzimisios, P., Boucouvalas, A. C., and Vitsas, V. (2003, December). IEEE 802.11 packet delay-a finite retry limit analysis. In Global Telecommunications Conference, 2003. GLOBECOM'03. IEEE (Vol. 2, pp. 950954). IEEE.

[3] Colandairaj, J., Irwin, G. W., and Scanlon, W. G. (2007). Wireless networked control systems with QoS-based sampling. IET Control Theory and Applications, 1(1), 430-438.

[4] Foh, C. H., and Tantra, J. W. (2005). Comments on IEEE 802.11 saturation throughput analysis with freezing of backoff counters. IEEE Communications Letters, 9(2), 130-132.

[5] Li, X., Li, D., Wan, J., Vasilakos, A. V., Lai, C. F., and Wang, S. (2015). A review of industrial wireless networks in the context of industry 4.0. Wireless Networks, 1-19.

[6] Lian, F. L., Moyne, J., and Tilbury, D. (2002). Network design consideration for distributed control systems. IEEE Transactions on Control Systems Technology, 10(2), 297-307.

[7] Sassi, I., Gouin, A., and Thiriet, J. M. (2016). A Bayesian network for diagnosis of networked mobile robots. Risk, Reliability and Safety: Innovating Theory and Practice: Proceedings of ESREL 2016 (Glasgow, Scotland, 25-29 September 2016), 340.

[8] Simon, D., Song, Y. Q., and Aubrun, C. (2013). Co-design approaches to dependable networked control systems. John Wiley and Sons.

[9] Tian, G., Camtepe, S., and Tian, Y. C. (2016). A deadline-constrained 802.11 MAC Protocol with QoS differentiation for soft real-time control. IEEE Transactions on Industrial Informatics, 12(2), 544-554.

[10] Tinnirello, I., Bianchi, G., and Xiao, Y. (2010). Refinements on IEEE 802.11 distributed coordination function modeling approaches. IEEE Transactions on Vehicular Technology, 59(3), 1055-1067.

[11] Ziouva, E., and Antonakopoulos, T. (2002). CSMA/CA performance under high traffic conditions: throughput and delay analysis. Computer communications, 25(3), 313-321. 